Voix et Images

\title{
Pour un nouvel éclairage sur les Lumières au Québec
}

\section{Bernard Andrès}

Volume 16, numéro 1 (46), automne 1990

Les correspondants littéraires d'Alfred DesRochers

URI : https://id.erudit.org/iderudit/200880ar

DOI : https://doi.org/10.7202/200880ar

Aller au sommaire du numéro

\section{Éditeur(s)}

Université du Québec à Montréal

ISSN

0318-9201 (imprimé)

1705-933X (numérique)

Découvrir la revue

Citer cet article

Andrès, B. (1990). Pour un nouvel éclairage sur les Lumières au Québec. Voix et Images, 16(1), 138-145. https://doi.org/10.7202/200880ar d'utilisation que vous pouvez consulter en ligne.

https://apropos.erudit.org/fr/usagers/politique-dutilisation/ 


\section{Pour un nouvel éclairage sur les Lumières au Québec}

\section{par Bernard Andrès, Université du Québec à Montréal}

Les quatre ouvrages dont il sera ici question méritent à coup sûr de figurer dans notre "Bibliothèque du bicentenaire». Le premier n'est autre que cette livraison d'Études françaises intitulée "l'Esprit de la Révolution" ${ }^{1}$. Ce numéro rassemble une douzaine d'universitaires canadiens et français autour de l'esprit de liberté, d'égalité, de fraternité qui a soufflé sur la pensée et les pratiques du XVIII siècle, et, depuis, sur les nôtres, explique Josiane Boulab-Ayoub, responsable de la publication. Bien que célébrant le bicentenaire en cette terre d'Amérique où eurent lieu les préludes de la "Grande Révolution", et, comme sa répétition générale, ce numéro s'attache d'abord et presque exclusivement aux aspects français ou européens de ce bouleversement considéré surtout sur le plan intellectuel. C'est d'une révolution culturelle qu'il s'agit ici, de son être symbolique, ontologique et social, de ses prémisses dans la pensée des. Philosophes à ses réalisations dans la praxis et le discours révolutionnaires et, au-delà, à ses effets ultimes sur la révolution philosophique allemande (cf. l'article de Jacques Aumètre sur Hegel). Ce cheminement du Sujet au Citoyen, les collaborateurs le retracent dans les écrits de Rousseau sur l'économie politique (Marcel Hénaff); de d'Holbach, Rousseau, Locke et Condillac sur les concepts de Bonheur, de Bienfaisance et de Vertu (Josiane Boulad-Ayoub) et de Progrès dans les débats sur l'instruction publique (Michel Grenon). Sur l'apport de la pensée et de l'histoire américaines, dont Josiane Boulad-Ayoub note bien qu'elles confortent le discours révolutionnaire, un seul article: celui de Louise Marcil-Lacoste, consacré à Thomas Paine dont le fameux pamphlet "Common Sense" parut en 1776 dans le Pennsylvania Magazine. 
Deux textes retiennent particulièrement l'attention dans cet ensemble d'analyses rigoureusement menées et documentées: ils concernent des cas limites de "dérapage » du discours révolutionnaire chez Sade (Jean Terrasse) et Marat (Michaël Lachance). On lit aussi avec un intérêt soutenu les contributions de Linda $A$. Lapointe et de Claudette Hould sur les beaux-arts et l'image (contre-)révolutionnaires. C'est à ces deux auteures qu'on doit aussi le support iconographique souvent inédit de ce superbe numéro ${ }^{2}$.

Il fallait s'attendre à ce que le bicentenaire suscite, de ce côté-ci de l'Atlantique, un regain d'intérêt pour une époque quelque peu négligée de l'histoire québécoise: histoire politique, histoire culturelle, histoire littéraire aussi bien. Que s'est-il écrit depuis plus d'un siècle à propos de la Révolution française et du Québec? Sur la cinquantaine de livres et d'articles recensés par Serge Leroux en $1989^{3}$, la moitié des titres sont postérieurs à 1960 (ceux de Claude Galarneau, Michel Grenon, John Hare, Fernand Ouellet, Pierre Tousignant, Jean-Pierre Wallot). Six d'entre eux paraissaient dernièrement, dont trois pour la seule année passée (il en sera principalement question dans ces pages): les actes partiels du $6^{\mathbf{e}}$ colloque du Centre interuniversitaire d'études européennes sur "Le Canada et la Révolution française", un autre collectif paru chez HMH, l'Image de la Révolution française au Québec. 1789-1989 et une monographie sur Valentin Jautard ${ }^{4}$. On ne peut que se réjouir de ce renouveau des recherches, puisque les travaux de NarcisseEutrope Dionne remontent quand même à 1905 et ceux de Ralph Flenley, Ivanhoé Caron et Lionel Groulx aux années 1920-1940. - Marcel Trudel publiait son Influence de Voltaire au Canada en 1945, Mason Wade ses Canadiens français en 1950. Les ouvrages de Brunet sur l'époque datent des années 1950-1960. Chacun à sa façon s'est interrogé sur l'impact de cette révolution sur les mentalités et les institutions de l'époque. Les présupposés théoriques et idéologiques nous apparaissaient à l'occasion, mais restaient le plus souvent implicites. Les sources de Narcisse-Eutrope Dionne provenaient notamment des historiens français contre-révolutionnaires. Tout en s'intéressant aux données socio-économiques et culturelles, l'abbé Caron cherchait avant tout à exalter le rôle salvifique du clergé (pour reprendre l'expression de Maurice Lemire). De même pour l'abbé Groulx. En 1945, l'anti-voltairisme anime encore la recherche de Trudel, autant que l'esprit anti-Canadian nourrit celle de Brunet en 1954. La différence majeure entre ces travaux - au demeurant incontournables - et les plus récentes publications ne réside pas dans l'«objectivité" de ces dernières, mais dans leur propension à mieux cerner leur lieu et leurs contraintes d'énonciation (aux plans institutionnel, idéologique, méthodologique). Si la plupart des chercheurs considèrent désormais favorablement les Lumières et la Révolution, s'ils s'avèrent plus critiques à l'égard de l'appareil clérical 
au $\mathrm{XIX}^{\mathrm{e}}$ siècle, plus sereins par rapport au(x) nationalisme(s), ils ne font pas de leurs opinions personnelles le sujet même de leur propos. Ils se situent plus explicitement dans les grands débats historiographiques et déplacent volontiers l'objet de l'événement proprement dit (la Révolution) à ses répercussions dans l'imaginaire et dans les discours d'accompagnement.

Du colloque de 1987 sur "Le Canada et la Révolution françaìse" 5 , Pierre H. Boulle et Richard A. Lebrun n'ont retenu que onze textes qui touchent principalement aux retombées intellectuelles, politiques et institutionnelles de la Révolution française sur l'histoire canadienne $d u X I X^{e}$ siècle. Après la présentation générale de l'historiographie européenne sur la Révolution, par Michel Vovelle, Jean-Pierre Wallot évoque la création des deux premiers mythes canadiens: la "Conquête providentielle" et les deux "races" fondatrices. Ces constructions, fondées pour une bonne part en réaction contre la Révolution, éclipseront bien vite celle-ci des esprits et des écrits subséquents:

C'est comme si la Révolution française n'existait pas ou, du moins, n'avait influé que très peu ou pas du tout sur la société bas-canadienne. Étrange contraste entre l'historiographie et les préoccupations des contemporains. (p. 15)

Wallot explique cette absence relative par une situation où un peuple conquis et francophone, majoritaire dans une colonie britannique, hérite d'un système parlementaire qu'il croit vraiment représentatif. Il met en relief l'accueil d'abord favorable des élites comme des " masses ", jusqu'à l'exécution des souverains français. Il apparaît que même après celle-ci l'agitation subsiste entre 1793 et 1797 , ce que confirmera dans le détail l'étude fort documentée de Mario Lalancette sur les mouvements populaires à la Malbaie. Cet écho positif de la Révolution fait aussi l'objet de l'article de Jean-Paul de Lagrave consacré à l'imprimeur Fleury Mesplet. On sait la position stratégique occupée par la Gazette du commerce et littéraire de Montréal dans la diffusion des Lumières au Québec. De Lagrave y consacrait une magistrale monographie en $1985^{6}$. Roland le Huenen examine pour sa part les variations $[d u]$ spectre révolutionnaire dans les premières manifestations littéraires canadiennes. Il signale ainsi que le premier poème sorti des jeunes presses québécoises fut une épître de Voltaire adressée au cardinal Querini (dans la Gazette de Québec du 23 mars 1767). Désireuses de miner le catholicisme, les autorités britanniques ne pouvaient alors qu'encourager la propagation des idées libérales. L'anglomanie bien connue des Philosophes ravissait l'occupant lorsqu'il la retrouvait diffusée par la Gazette de Québec en 1791, dans un poème commémoratif du 14 juillet 1789:

De l'esclavage, enfin, les chaînes sont brisées;

Nous admirons l'Anglois, il est plus qu'imité! 
Le tournant s'effectue en 1793 , à l'annonce de l'exécution du roi. Un nouvel usage de la Révolution voit le jour: il s'agit de la condamner sans réserve pour mieux prouver son loyalisme à Albion, alors entrée en guerre. L'inversion sémiotique bat son plein dans le discours religieux où l'esprit des Lumières se voit changé en prince des ténèbres, et l'ennemi d'hier, l'Anglais' conquérant, en l'ami d'aujourd'hui. Seuls des républicains comme Fleury Mesplet, Valentin Jautard, Henri Mézière, des réformistes comme Pierre Du Calvet, tous sympathiques à la cause américaine, restent fidèles à leurs convictions, certains payant de leur personne dans les cachots de Haldimand. L'arbitraire du prince sera relayé par l'appareil judiciaire naissant, qui louvoie entre les traditions britannique et française. Les pressions des Loyalistes et le rôle des élites juridiques dans la répression des émeutes à la fin du siècle sont étudiés par Louis A. Knafla. Les articles de Bernard Plongeron sur "Le vécu religieux des populations dans l'Europe révolutionnaire", de Luca Codignola sur les relations entre Rome, Paris et Québec et de Gilles Chaussé sur l'Église canadienne au tournant du XVIII ${ }^{e}$ siècle permettent de situer le Québec dans une perspective comparatiste. Aux confins d'une théologie révolutionnaire, certains courants de la pensée religieuse européenne battent en brèche la vison d'une Église monolithique et réactionnaire. Sans parler d'un plus grand libéralisme dans l'Église nord-américaine, il appert que l'éloignement des anciennes métropoles favorise alors une certaine autonomisation des institutions religieuses et, au Québec, le progrès des idées de La Mennais au début du XIX ${ }^{e}$ siècle.

La dernière section de cet ouvrage porte sur l'écho différé de la Révolution, tel qu'il se manifeste dans le Code civil québécois (Richard Larue) et dans la presse anglophone et francophone au XIX ${ }^{\mathrm{e}}$ siècle. Anthony Rasporich analyse ainsi la couverture canadienne des révolutions françaises de 1848 et 1870-1871 : devant la montée du socialisme sous la Commune, la Révolution bourgeoise de 1789 apparaît infiniment moins menaçante aux élites canadiennes. Trois phases marquent donc les lendemains de la Révolution française au Québec, comme le note en conclusion Pierre H. Boulle: a) de 1789 à 1792-93, c'est un peu l'âge d'or de la diffusion des Lumières ${ }^{7}$, tant chez les élites qu'en milieu rural où la population semble beaucoup mieux informée au Canada qu'elle ne l'est en France même; b) de 1793 à 1815 , avec l'entrée en guerre de l'Angleterre, c'est le rejet de l'expérience française dont les excès génèrent le mythe de la Conquête providentielle; c) de la paix de 1815 à la fin du siècle, c'est l'époque du souvenir ravivé par 1837-1838 et marquée par l'extinction de la féodalité, le cheminement parfois difficile des idées libérales.

On retrouve nécessairement ces trois phases dans le second ouvrage, l'Image de la Révolution française au Québec. 1789- 
1989, dont le maître d'œuvre est Michel Grenon. Tout aussi intéressant que le précédent, ce livre l'excède par la période et par certains aspects le champ d'investigation. Au-delà des redites toujours inévitables (pour les décennies 1790-1830 notamment ${ }^{8}$ ), on retient ici l'accent mis sur les représentations de la Révolution telles qu'elles ont évolué sur la longue durée: importation d'une image, variations sur le motif, déconstruction et réutilisations successives de ce matériau idéologique, de la Constitution de 1791 à la Révolution tranquille, en passant par $1837-38^{9}$, l'Institut canadien, $1889^{10}$, le Congrès eucharistique de 1910, le Refus global, la Commission Parent et la Crise d'Octobre 1970. La Révolution comme image mentale, mais aussi comme thème littéraire ou document iconographique ${ }^{11}$. En effet, aux analyses historiques et philosophiques (Michel Grenon, Marcel Trudel, Claude Galarneau, Jean-Pierre Wallot, Pierre Savard, Gilles Chaussé), se greffent des considérations sur les lettres (Réginald Hamel) et les arts québécois (Claudette Hould, Laurier Lacroix, François-Marc Gagnon ${ }^{12}$ ).

Dans le prolongement de ses nombreux travaux sur l'époque, Marcel Trudel choisit l'épisode de l'université «neutre» dont le projet avorta justement au tournant de 1789 . Son analyse du conflit qui opposa Mgr Hubert au coadjuteur Bailly de Messein à propos de cette institution d'enseignement supérieur que les Anglais songeaient à promouvoir aboutit à une condamnation du coadjuteur qui avait osé affronter publiquement son évêque. Un membre du clergé qui prône tolérance et laïcisme, qui déplore que les sciences languissent sous le fetfa de l'ignorance et le lacet du despotisme 13 ! Trudel reprend curieusement à son compte les reproches traditionnellement adressés au (trop) brillant curé de Pointe-aux-Trembles: mauvais esprit, arrivisme, cupidité, insubordination, méchanceté, etc. C'est peut-être que ce clerc récalcitrant formé à Paris était guidé par cet esprit des Lumières peu apprécié par Trudel qui lui préfère de loin le libéralisme à l'anglaise: le vent souffle aussi de l'Angleterre, dit-il (surtout, laisse-t-il entendre, réduisant l'Encyclopédisme à un épiphénomène généré par l'anglophilie des Philosophes!). Claude Galarneau rétablit heureusement l'équilibre en examinant l'apport de la France au Québec en matière d'immigration, même après la Conquête. Peu de nouveaux venus entre 1763 et 1789 (une vingtaine), mais quelles personnalités si l'on songe parmi les quatre médecins à Pierre de Sales Laterrière; des peintres aussi, des musiciens, un poète et dramaturge comme Joseph Quesnel, l'imprimeur Fleury Mesplet à qui l'on doit la Gazette du commerce et littéraire de Montréal, un journaliste comme Valentin Jautard. C'est après 1792 que le BasCanada connut son lot de réfugiés contre-révolutionnaires de la noblesse ou du clergé. Il y eut même un faux Louis XVII qui se fixa près de Berthier, mais moins d'une cinquantaine de prêtres sur les 8000 passés en Angleterre s'établirent ici. On sait leur rôle dans le 
raffermissement de l'Église québécoise et, plus largement, sur les mentalités pendant plus d'un siècle. Ce qu'on connaissait moins, c'est l'apport de certains d'entre eux dans le domaine des lettres et des arts. Pour ce qui concerne la peinture, Laurier Lacroix rappelle l'étrange affaire des "tableaux Desjardins", pieuses épaves sauvées de l'hydre révolutionnaire, acheminées au Québec entre 1817 et 1820, et dont la présence massive bouche [...] l'horizon des peintres québécois du XIX $X^{e}$ siècle (Michel Grenon).

Réginald Hamel signe la seule étude du volume consacrée à la littérature québécoise dans ses rapports à la Révolution française. "Paroles gelées et idées gelées", titre-t-il pour souligner le peu d'impact des idées libérales sur la production littéraire jusqu'à la Révolution plus que tranquille. Considérant les périodiques de 1764 à 1823 (notamment la première série du Canadien) et le récit de Frances Brooke, The History of Emily Montaigu (1769), il dégage un portrait intellectuel de l'époque au tournant du siècle. Le relevé précis des auteurs français et anglais diffusés par ces textes ne permet nullement de discerner un courant évident d'œuvres littéraires se rattachant à la Révolution. Toute forme de républicanisme semble vouée à l'échec, contrairement à ce que soutenait Joseph Costisella en 1968 (Hamel lui reproche un échantillonnage trop limité d'auteurs et une acception trop large des termes révolution ou révolutionnaire). L'examen qui suit porte sur un corpus allạt de Quesnel à Chauveau. Mézière, Bibaud, Aubert de Gaspé, Angers, Lacombe et Chauveau confirment dans l'ensemble l'impression de Hamel: les Canadiens français sont devenus des velléitaires. Seul Doutre (impliqué plus tard dans l'affaire Guibord) aurait pu inquiéter les bonnes âmes des années 1840-1850. On retrouve bien à l'Institut canadien des traces atténuées du discours révolutionnaire, mais l'artillerie lourde de $\mathrm{Mgr}$ Bourget en vient vite à bout. De Chevalier à Girard les cancrelats cafardent, alors qu'au $\mathrm{XX}^{\mathrm{e}}$ siècle, on passera de la dictature des bedeaux à la dictature du prolétariat, résume avec son sens de la formule l'auteur dont nous ne résistons pas à citer la conclusion (faute de pouvoir ici mieux rendre justice au détail de l'analyse):

La société québécoise est devenue comme une folle enveloppée dans les loques du drapeau de Carillon, ayant perdu son "je» [...] dans les corridors de l'histoire, et qui va, un roman Harlequin à la main, hurlant aux croisées: "Ne sait point et ne veut rien sawoère (sic)... y'a rien là ", derniers échos sonores des vaincus de 1759 !...

L'apport de Valentin Jautard au milieu intellectuel québécois n'a été souligné qu'au hasard des notices biographiques ou de remarques sur les débuts du journalisme. Associé à Fleury Mesplet dont il fut le rédacteur principal à la Gazette du commerce et littéraire (1778- 
1779), il a peut-être pâti de l'anonymat relatif où le plongeait son pseudonyme: le Spectateur tranquille. Les autorités de l'époque ne s'y trompèrent pourtant pas, lui infligeant, ainsi qu'à l'imprimeur, trois ans de prison sans procès, histoire de faire goûter aux Philosophes montréalistes les vertus de la justice britannique. La plupart des commentateurs se sont égayés de leur mésaventure, traitant bien à légère le premier cas de répression manifeste de la liberté d'expression sous le Régime anglais ${ }^{14}$. Les jugements les plus négatifs, les appréciations les plus biaisées, les moins sûres des informations ont circulé pendant près d'un siècle sur ces pionniers, sans qu'historiens ni critiques ne prennent la peine de remonter aux sources - ce qu'entreprennent enfin Jean-Paul de Lagrave et Jacques G. Ruelland dans Valentin Jautard. 1736-1787. Premier journaliste de langue française au Canada. L'intérêt de cette monographie repose autant sur ses mises au point bio- et bibliographiques, que sur la présentation commentée des idées défendues par Jautard. S'y ajoute la dimension anthologique de l'ouvrage, puisque la section la plus importante sur "L'œuvre journalistique" (près des deux tiers de l'ensemble) consiste en une reprise des articles dont il est assuré qu'ils sont de la plume de Jautard ou de ceux qui l'interpellaient, et qui rendent plus facile la compréhension des réponses données par le journaliste (p. 102). On a ainsi, dans l'ordre chronologique de parution, une vision dynamique des échanges intellectuels à l'époque de la fondation de l'Académie de Montréal. Les «Lettres de prison " témoignent ensuite du drame vécu par le journaliste et l'imprimeur et de leur détermination face à l'arbitraire du général Haldimand ${ }^{15}$. Jautard sortira brisé d'une épreuve dont ses compagnons d'infortune Laterrière et Mesplet sauront mieux se relever.

À ce propos, il faut signaler que le principal témoignage de cet emprisonnement fut celui de Pierre de Sales Laterrière (1743-1815), dans ses Mémoires parus à titre posthume en $1873^{16}$. Or, il se trouve que ce dernier n'entretenait pas les meilleures relations avec ses codétenus. Le portrait qu'il nous en laisse et dont la critique s'est largement inspirée n'est pas des plus flatteurs (pour user de la litote). De Lagrave et Ruelland se sont donc attachés à le corriger. Mais ce faisant, ils n'ont pu que charger à leur tour la figure de Laterrière avec un parti pris manifeste contre cette autre victime de Haldimand qui leur sert trop commodément de faire-valoir (p. 29-46). Il eût été plus décent de pondérer les jugements de Laterrière en les attribuant aux inévitables frictions causées par la promiscuité forcée de trois hommes aux caractères tranchés, mais aussi aux défaillances mémorielles du septuagénaire qui n'entreprit que trente ans plus tard et à des fins intimes le récit de cette épreuve ${ }^{17}$. Avec $\mathrm{Du}$ Calvet, Mesplet, Jautard, Mézière et Quesnel, Laterrière reste une des personnalités marquantes de cette fin du XVIII ${ }^{e}$ québécois en prise 
directe sur le monde. Nul doute que d'autres publications contribueront bientôt à relayer celles du bicentenaire, jetant un nouvel éclairage sur les Lumières au Québec.

1 "L'Esprit de la Révolution", Études françaises, vol. XXV, $n^{08} 2 / 3$, automne 1989, 291 p.

2 Claudette Hould est également responsable du remarquable catalogue du Musée du Québec intitulé l'Image de la Révolution française, Québec, Publications du Québec et Éditions du Musée du Québec, 1989, 450 p. (édition anglaise: Images of the French Revolution).

3 Au bilan historiographique de Josiane Boulad-Ayoub dans Études françaises, il faut ajouter pour le Québec la bibliographie commentée de Serge Leroux dans l'Image de la Révolution française au Québec. 1789-1989, sous la direction de Michel Grenon, Montreal, Hurtubise HMH, 1989, p. 229-249.

4 Le Canada et la Révolution française, sous la direction de Pierre $\mathrm{H}$. Boulle et Richard A. Lebrun, Actes du $6^{\mathbf{e}}$ colloque du CIEE (1987), Montreal, Centre interuniversitaire d'etudes européennes, 1989, 183 p.; Valentin Jautard (1736-1787). Premier journaliste de langue française au Canada, de Jean-Paul de Lagrave et Jacques G. Ruelland, Sainte-Foy, le Griffon d'argile, 1989, 390 p. Signalons également le colloque organisé a l'automne dernier par le Centre de recherches en civilisation canadienne-française de l'Université d'Ottawa (actes à paraitre).

5 Colloque tenu en octobre 1987 à l'Université de Montréal, sous les auspices de la Société historique du Canada, de l'Institut d'histoire de l'Amérique française (IHAF) et du Centre interuniversitaire d'études européennes (CIEE).

6 Jean-Paul de Lagrave, Fleury Mesplet (1734-1794): diffuseur des Lumières au Québec, Montréal, Patenaude éditcur, 1985, 504 p. Il s'agit là d'un modèle de recherche, de documentation et d'analyse, incontournable pour quiconque travaillerait sur le dernier quart du XVIII siecle québécois.

7 Au prix néanmoins d'emprisonnements aussi arbitraires que ceux dont pâtissent alors Mesplet, Jautard, Du Calvet et Laterrière...

8 Gilles Chaussé qui collabore aux deux livres reprend grosso modo les mêmes données dans le second, clargissant toutefois l'analyse au XX $\mathrm{XX}^{\mathrm{e}}$ siecle.

9 Il faut lire l'analyse très fouillée que donne Jean-Pierre Wallot des jeux d'influence, de diffraction et de réfraction qui s'opèrent entre les Lumières, la Révolution américaine et la Révolution française, de 1789 à 1838 (p. 61-104).

10 Pierre Savard dresse un bilan fort éclairant de ce centenaire qui n'a pas eu lieu (p. 105-121).

11 Lire à ce sujet l'étude de Claudette Hould sur "La gravure révolutionnaire et son impact sur les consciences", p. 173-182.

12 - Ce dernier considère les aléas du terme "révolution" dans les manifestations automatistes des annces 1945-50, p. 201-218.

13 Je tire cette citation de Bailly de son mémoire du 5 avril 1790, reproduit par Yvon André Lacroix dans les Écrits du Canada français, $n^{\circ} 28,1969$, p. 241 (les gras sont de l'auteur).

14 La troisième section est un bilan sévère de la critique et de la tradition historiographique à propos de Jautard (de Casgrain à Rumilly, en passant par Sulte, Roy, Groulx, Wade, Marion, Trudel, Brunct, etc.).

15 De ces emprisonnements arbitraires entre 1779 et 1783 , témoigne l'Appel à la justice de l'État, de Pierre Du Calvet, dont de Lagrave et Ruelland ont aussi publié de larges extraits aux éditions le Griffon d'argile, en 1986.

16 Mémoires de Pierre de Sales Laterrière et de ses traverses, édition intime, Québec, Imprimerie de "l'Événement », 1873, in-12, 272 p.

17 Une réhabilitation de cette figure marquante des lendemains de Conquête s'impose. L'auteur de ces lignes prépare actuellement une biographie critique de Pierre de Sales Laterrière. 